\title{
Treatment Satisfaction and Burden of Illness in Patients with Newly Diagnosed Multiple Myeloma
}

\author{
Robert M. Rifkin ${ }^{1}$ Jill A. Bell ${ }^{2}$ Pronabesh DasMahapatra ${ }^{3} \cdot$ Michael Hoole $^{4} \cdot$ Maria Lowe $^{4} \cdot$ Chris Curran $^{4}$. \\ Scott Campbell ${ }^{2} \cdot$ Peijie Hou $^{2} \cdot$ Dorothy Romanus $^{2}$
}

Published online: 11 October 2019

(c) The Author(s) 2019

\begin{abstract}
Objectives This observational study identified attributes of patient-reported satisfaction with therapy for multiple myeloma (MM), described the treatment-related time burden and indirect costs, and investigated the effect of administration route (oral vs. injectable) on these outcomes among patients with newly diagnosed MM (NDMM) and among caregivers.

Methods Patients residing in the USA with a self-reported diagnosis of NDMM were recruited from PatientsLikeMe, MyelomaCrowd, and Facebook (16 December 2016 and 6 July 2017) to complete an electronic survey including questions on treatment experience, economic burden, and standardized patient-reported outcome measures, including the Treatment Satisfaction Questionnaire for Medication with three domains (global satisfaction, effectiveness, and convenience) and the Work Productivity and Activity Impairment Questionnaire. Univariate and multivariate analyses identified predictors of patient-perceived treatment satisfaction.

Results Among 188 patients, worse Eastern Cooperative Oncology Group performance status (ECOG PS) was correlated with lower patient-perceived effectiveness and convenience of their current treatment. White race and oral administration route were independently correlated with higher patient-perceived convenience of treatment. Injectable therapy use was associated with a trend towards increased activity impairment ( 43 vs. $34 \% ; p=0.05$ ) and significantly higher time burden of treatment administration, with threefold higher adjusted indirect costs of MM therapy compared with solely orally administered therapies (monthly mean \$US482 vs. 153; 2016 values; $p<0.0001$ ).

Conclusions Factors associated with patient-perceived satisfaction with NDMM treatment-ECOG PS, race, administration route-warrant increased attention in shared treatment decision making to help identify patient needs and improve the patient's treatment experience. The use of orally administered therapies could improve patients' activity impairment and reduce the time burden associated with therapy.
\end{abstract}

Pronabesh DasMahapatra is affiliated with PatientsLikeMe at time of study.

Electronic supplementary material The online version of this article (https://doi.org/10.1007/s41669-019-00184-9) contains supplementary material, which is available to authorized users.

Dorothy Romanus

Dorothy.Romanus@takeda.com

1 US Oncology/Rocky Mountain Cancer Centers, Denver, CO, USA

2 Millennium Pharmaceuticals, Inc., a wholly owned subsidiary of Takeda Pharmaceutical Company Limited, 40 Landsdowne Street, Cambridge, MA 02139, USA

3 Sanofi, Bridgewater, NJ, USA

4 PatientsLikeMe, Cambridge, MA, USA

\section{Introduction}

Multiple myeloma (MM) is a progressive haematologic malignancy. In the USA, in the last decade, the incidence of newly diagnosed MM has risen by approximately $0.9 \%$ each year [1]. In 2018, an estimated 30,770 Americans were diagnosed with MM, which accounts for $1.8 \%$ of all new cancer cases in the USA and $2.1 \%$ of all cancer deaths [1]. With the development of novel therapies and a better understanding of the biology of MM, mortality from MM has decreased by approximately $0.5 \%$ each year from 2006 through 2015 in the USA, and more patients are achieving lasting remission [1-3].

Management of newly diagnosed MM (NDMM) is challenging, given the heterogeneity of the disease and the plethora of newly available treatment combinations. Patients diagnosed with active (symptomatic) MM are treated with 


\section{Key Points for Decision Makers}

This study suggests that attributes such as better Eastern Cooperative Oncology Group performance status and oral treatment administration route lead to higher patient perceptions of satisfaction with treatment for newly diagnosed multiple myeloma (NDMM), whereas non-White patients report lower satisfaction.

Oral administration route was also associated with less time spent receiving treatment, lower activity impairment, and reduced economic burden for patients.

Increased attention to these factors in shared treatment decision making is warranted to help identify individual patient needs, preferences, and expectations for NDMM treatments.

frontline induction therapy, followed by high-dose chemotherapy and autologous stem cell transplant in eligible patients, and maintenance therapy to prevent relapse and prolong remission through continued treatment. Options for therapy include combinations of proteasome inhibitors (bortezomib, carfilzomib, ixazomib), immunomodulatory drugs (lenalidomide, thalidomide, pomalidomide), monoclonal antibodies (daratumumab, elotuzumab), alkylating agents, and steroids combined as doublets and triplets [4]. Choice of initial combination therapy can be influenced by prognostic factors following evaluation of the biological subtype of disease, patient-related factors such as impaired performance status (PS), living/working situation, comorbidities (as patients are usually elderly and frail), and treatment side effects.

Currently, the number of clinical trials in NDMM directly demonstrating the superiority of one regimen over another in terms of patient quality of life is limited. Clinical efficacy, toxicity, and quality of life notwithstanding, additional factors must also be considered when determining the specific course of treatment at the individual patient level. In particular, patient satisfaction, a patient-reported outcome (PRO) that provides insights into the patient's perspective on their current treatment experience, may be useful for differentiating among alternative treatments [5].

Studies in patients with various malignancies indicate that PROs reflect a patient's treatment experience, which is influenced by route of treatment administration (oral vs. parenteral), costs, time burden, convenience, and impact of therapy on quality of life [6-9]. A recent review of the administration preferences of patients with cancer, specifically between oral and intravenous treatment, as well as the factors contributing to preference, demonstrated that patients with cancer preferred oral treatment over intravenous administration of therapy because of convenience, perception of efficacy, and past experience [7]. Generally, patients with cancer were unwilling to accept reduced efficacy or increased toxicity in favour of other treatment attributes, such as convenience [7]. However, in the breast cancer setting, where anticancer therapy is dominated by intravenous chemotherapy, patients showed a high acceptance of oral anticancer therapy and were willing to tolerate an increased risk of certain side effects in exchange for an oral regimen [10-12].

Patient satisfaction with treatment is associated with adherence to therapy, willingness to continue treatment, preventive care, healthcare resource use, care delivery, objective measures of health outcomes, and safety of therapy across a wide range of disease areas, patient populations, and outcome measures [13-16]. Therefore, the patient experience is increasingly used as a criterion to evaluate quality in healthcare [13].

Published studies reporting on the determinants of patient satisfaction specific to NDMM therapy are lacking. The objectives of the current observational study were to identify factors associated with patient-reported satisfaction with MM therapy, describe the treatment-related time burden and indirect costs, and investigate the effect of administration route (oral vs. injectable) on patient-reported satisfaction with treatment and time and financial burden among patients with NDMM and their caregivers. An improved understanding of the experiences of patients with NDMM may help facilitate individualized treatment decision making for such patients.

\section{Patients and Methods}

\subsection{Study Design and Participants}

In this cross-sectional study conducted between 16 December 2016 and 6 July 2017, patients with a self-reported diagnosis of NDMM completed a self-administered electronic survey (see the Electronic Supplementary Material [ESM]) investigating their treatment experience and burden of illness. Patients were recruited from the research-ready community of PatientsLikeMe (PLM; www.patientslikeme. com), the patient advocacy group MyelomaCrowd, and posts distributed via the Takeda Oncology Facebook page. Interested patients received a link to the survey via email, private message, or social media.

Patients were eligible to participate in this study if they were aged $\geq 18$ years, residing in the USA, and currently receiving treatment for NDMM. Patients were classified as having NDMM if they reported never changing their treatment due to disease progression or recurrence. Patients 
were excluded if they had concomitant amyloidosis or had been diagnosed with and treated for other cancers in the past 5 years. The study received ethical approval from the New England Institutional Review Board. Patients provided informed consent electronically before completing the survey. Respondents were not remunerated for their participation.

\subsection{Patient-Reported Measures}

The patient self-reported survey recorded eligibility criteria, demographic characteristics, treatment background and satisfaction, patient and caregiver time burden associated with MM therapy, and patient work and activity impairment. The survey included the following PRO measures: Treatment Satisfaction Questionnaire for Medication (TSQM-9) [17], an adapted patient-reported version of the Eastern Cooperative Oncology Group performance status (ECOG PS) [18], and the Work Productivity and Activity Impairment Questionnaire: Specific Health Problem V2.0 (WPAI:SHP) [19].

The TSQM-9 is a generic measure that assesses treatment satisfaction with medication [17, 20]. The TSQM-9 includes nine items scored on a 5- or 7-point Likert-type scale that cover three domains (global satisfaction, effectiveness, and convenience) corresponding to distinct aspects related to patient satisfaction with their treatment during the previous 2-3 weeks. Scores range from 0 to 100 , and higher scores on the TSQM-9 domains indicate higher global satisfaction, better perceived effectiveness, and better convenience [17].

The adapted patient-reported version of the ECOG PS consists of a single item evaluating the current PS of oncology patients [18]. ECOG PS is widely used to quantify functional status and determine prognosis in oncology [21]. ECOG PS describes functional impairment at the patient level, a patient's ability to participate in self-care and daily activities, and their physical ability. The single item is a question that includes five numerical responses scored on a scale ranging from 0 to 4 , with 0 denoting fully active without restriction and 4 defined as completely disabled and unable to carry out any self-care. In general, an ECOG $<3$ indicates predominantly good PS [18].

The WPAI:SHP measures absenteeism (work time missed), presenteeism (impairment at work), work productivity loss (absenteeism plus presenteeism), and activity impairment and was adapted specifically for MM. In the current patient survey, the WPAI:SHP included one question on current employment status, two questions that assessed the number of hours missed from work due to $\mathrm{MM}$, one question on the number of hours actually worked, one question on the impact of MM on work productivity, and one question that assessed the impact of MM on non-workrelated daily activities. The degree to which MM affected absenteeism, presenteeism, and work productivity loss was measured in employed patients. The degree to which MM affected non-work-related activities was measured in all patients regardless of employment status. WPAI:SHP outcomes are expressed as impairment percentages, with higher numbers indicating greater impairment and less productivity, i.e. worse outcomes.

In addition to the PROs, the survey included customized MM therapy-related time burden questions describing the monthly number of MM-related treatment visits, the number of visits accompanied by a caregiver, the average time to get to the treatment visit, and the average amount of time spent at the treatment visit.

\subsection{Analysis}

Continuous variables are summarized as means \pm standard deviations (SDs) and medians (interquartile range), whereas categorical measures are summarized as counts and percentages. Primary outcomes of interest corresponding to each of the three domains of the TSQM-9 and characteristics associated with greater patient perception of global satisfaction, treatment effectiveness, and convenience (TSQM-9 domains) were assessed in univariate models.

To identify factors independently associated with each of the three domains of the TSQM-9, variables of interest, and those with a $p<0.1$ in univariate analysis of the outcome of interest, were assessed using general linear models with a stepwise selection algorithm with an entry and retention $p$ value cut-off of 0.05 after adjusting for prior treatment history (prior treatment experience [injectable only vs. any oral therapy exposure vs. no prior therapy], stem cell transplant history). The following potential factors were considered in the model selection: age (continuous), race (non-White vs. others), ECOG PS (0-1 vs. $\geq 2)$, number of agents in current therapy ( 1 vs. 2 vs. $\geq 3$ ), current treatment administration mode (oral [oral] vs. injectable with or without oral [injectable]), and monthly patient time spent receiving therapy (travel and doctor's visit time). Interactions between current treatment administration mode and monthly patient time spent receiving therapy and between number of agents and administration mode in current therapy were tested for each outcome but were not significant.

The effect sizes on the TSQM-9 domain are calculated using Cohen's $f^{2}$ [22]. The global effect size is defined as the ratio between the proportion of variation in dependent variables explained by the independent variables and the unexplained variation. In addition, a variation of Cohen's $f^{2}$ was used to measure the local effect size of each individual independent variable in the multivariate analysis, which reflects the variance uniquely explained by the variable of interest while accounting for other variables. According to Cohen's guideline, the thresholds for defining a small, 
medium, and large effect size are $f^{2} \geq 0.02, f^{2} \geq 0.15$, and $f^{2} \geq 0.35$, respectively.

Time burden was calculated based on responses to MM therapy-related time burden questions in the patient selfreported survey. Average travel time burden per month was defined as the number of doctor's visits per month multiplied by the average time for round-trip travel. Average time spent at a doctor's visit per month was defined as the number of doctor's visits per month multiplied by the average time spent at the visit(s). The total time burden placed on patients as a result of monthly doctor's visits was defined as the sum of the average travel time per month and the average time spent at the doctor's visit per month. The total time burden placed on caregivers as a result of monthly doctor's visits was defined as the number of doctor's visits accompanied by caregivers multiplied by the average time for round-trip travel plus average time spent at the doctor's visit. The value of patient time was applied to all patients regardless of employment type. Data pertaining to caregivers were obtained indirectly from patient reports. Monthly estimates of indirect treatment-related costs were obtained from publicly available data sources (Table 1) and combined with survey responses to determine the economic burden. Resource utilization and economic burden of illness outcomes were compared between patients currently receiving oral versus injectable therapy.

Monthly costs and time burden were compared by fitting generalized linear models (GLMs) with gamma distribution and a log link (with an addition of a small constant [+1] to every observation to account for zero values), and GLM with a Poisson distribution and a log link were used for comparison of number of doctor's visits per month. Patient work and activity impairment were analysed using general linear models. Multivariate analyses to adjust for confounders between resource utilization, costs, work/activity impairment outcomes, and current mode of administration were adjusted for age, ECOG PS, and prior stem cell transplant history. As an assessment of the goodness of fit for model fitting, we estimated ratios of scaled deviance/degrees of freedom. A ratio value of around one indicates that the model fits the data well. Values much larger than or smaller than one may indicate a poor fit of the data or over-/underdispersion of the response variable. All data analysis was conducted in SAS Enterprise Guide 9.4 (SAS Institute Inc., Cary, NC, USA). Unless otherwise stated, all analyses were 2-tailed with a significance level of 0.05 .

\section{Results}

A flow diagram showing patient selection for this study is provided in Fig. 1. Data from 188 patients with NDMM were included in the analyses.

\subsection{Baseline Characteristics}

The demographic characteristics of the included patients are summarized in Table 2 . The mean age was $61 \pm 8.92$ years, $61 \%$ were female, $87 \%$ were White, and $73 \%$ had an ECOG PS $0-1$. The majority of patients were not living alone (85\%), and 50\% had a college education or higher. Most patients reported having commercial medical (46\%) and commercial pharmacy $(61 \%)$ insurance coverage. One-third of the patients were employed (31\%), and as many as $22 \%$ reported being medically unable to work. The majority of patients were previously exposed to an orally administered treatment (63\%), and $48 \%$ had received a prior stem cell transplant. Most patients were treated at an academic centre
Table 1 Data sources used to evaluate economic impact of multiple myeloma treatment

\begin{tabular}{|c|c|c|}
\hline \multicolumn{3}{|l|}{ Economic estimates } \\
\hline Data & Estimated value (\$US) & Source \\
\hline Value of patient time & $23.86 / \mathrm{h}$ & $\begin{array}{l}\text { BLS national average wage } \\
\text { all occupations (2016) [23] }\end{array}$ \\
\hline Value of caregiver time & $\begin{array}{l}\text { 13.25/h (combined average of } \\
\text { three sources referenced) }\end{array}$ & $\begin{array}{l}\text { NCSL state minimum wages } \\
(2017) \text { [24] } \\
\text { BLS mean home health aide } \\
\text { wage (2016) [25] } \\
\text { Cost of care survey (2016) } \\
\text { [26] }\end{array}$ \\
\hline Per drive cost (self-drive) & $0.17 / \mathrm{mile}$ & IRS mileage rates (2017) [27] \\
\hline Per drive cost (other drive) & $0.54 / \mathrm{mile}$ & IRS mileage rates (2017) [27] \\
\hline Per travel cost (public transport) & 2.13/trip & APTA (2016) [28] \\
\hline Per travel cost (taxi/rideshare) & $\begin{array}{l}2.41 / \text { mile } \\
2.89 \text { (base fare) }\end{array}$ & TaxiFareFinder (2017) [29] \\
\hline
\end{tabular}

APTA American Public Transportation Association, BLS Bureau of Labor Statistics, IRS Internal Revenue Service, NCSL National Conference of state legislatures 


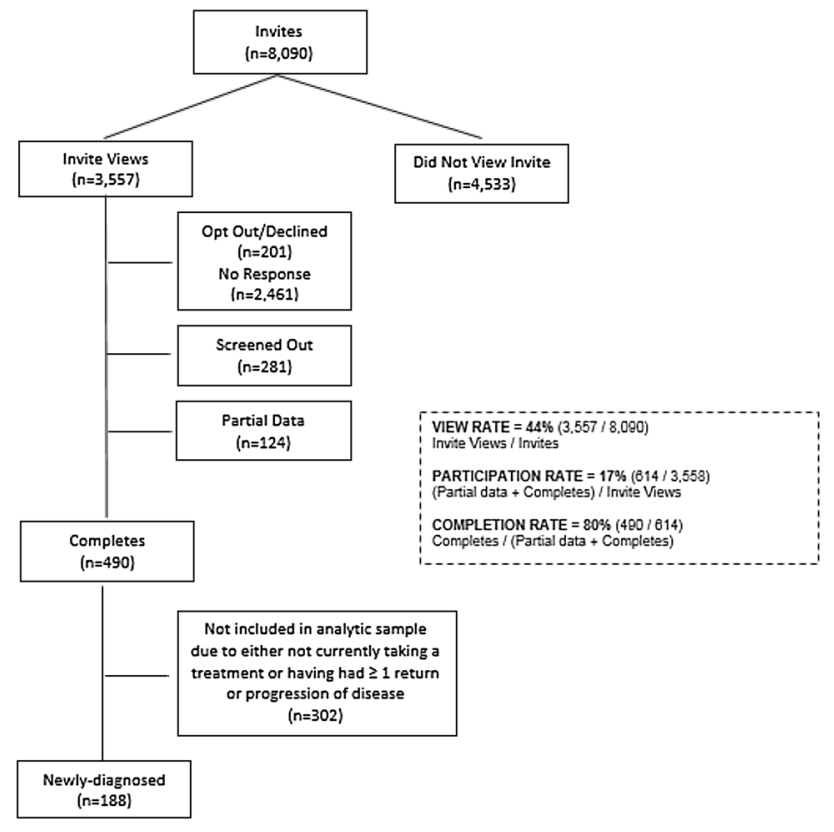

Fig. 1 Flow diagram of study sample

(80\%). Most patients (72\%) were receiving monotherapy or a doublet regimen as their current treatment.

\subsection{Predictors of Treatment Satisfaction- Treatment Satisfaction Questionnaire for Medication}

The results of the univariate analysis and final results of the multivariate models after adjustment for prior treatment experience (injectable only vs. any oral therapy exposure vs. no prior therapy) and stem cell transplant history are summarized in Tables S1 in the ESM and Table 3, respectively. In multivariate analyses, ECOG PS was the only factor associated with the patient-perceived effectiveness scale. Patients with an ECOG PS 0-1 had a 9.8-point higher score on the effectiveness scale than patients with an ECOG PS $\geq 2$ (Table $3 ; p=0.003$ ). Similarly, ECOG PS was significantly associated with the convenience scale; patients with an ECOG PS 0-1 had a 7.7-point higher score on the convenience scale than patients with an ECOG PS $\geq 2$ (Table 3; $p=0.007)$. Additional predictors of convenience included race (Table $3 ; p=0.003$ ) and administration route of current treatment (Table 3; $p=0.014$ ). Non-White patients had a 12.8-point lower score on the convenience scale than others (Table 3; $p=0.002$ ); patients receiving oral-only therapy had a 6.8-point higher score on the convenience scale than those receiving injectable with or without oral therapy. In tandem, the effect size for the three predictors of the convenience scale was moderate (global Cohen's $f^{2}=0.185$ ). Interestingly, after adjusting for prior treatment experience
Table 2 Patient and treatment characteristics among patients with newly diagnosed multiple myeloma receiving first-line therapy

\begin{tabular}{|c|c|}
\hline Characteristic & Total $(N=188)$ \\
\hline \multicolumn{2}{|l|}{ Age, years } \\
\hline Mean & $61.3 \pm 8.92$ \\
\hline Median (IQR) & $63.0(54.0-67.0)$ \\
\hline$<65$ & $111(59.0)$ \\
\hline $65-74$ & $68(36.2)$ \\
\hline$\geq 75$ & $9(4.8)$ \\
\hline \multicolumn{2}{|l|}{ Sex } \\
\hline Female & $114(60.6)$ \\
\hline Male & $73(38.8)$ \\
\hline Missing & $1(0.5)$ \\
\hline \multicolumn{2}{|l|}{ Race } \\
\hline Non-White & $18(9.6)$ \\
\hline White & $164(87.2)$ \\
\hline Missing & $6(3.2)$ \\
\hline \multicolumn{2}{|l|}{ Ethnicity } \\
\hline Hispanic & $5(2.7)$ \\
\hline Non-Hispanic & $174(92.6)$ \\
\hline Missing & $9(4.8)$ \\
\hline \multicolumn{2}{|l|}{ ECOG PS } \\
\hline $0-1$ & $138(73.4)$ \\
\hline$\geq 2$ & $50(26.6)$ \\
\hline \multicolumn{2}{|l|}{ Living situation } \\
\hline Alone & $29(15.4)$ \\
\hline Not alone & $159(84.6)$ \\
\hline \multicolumn{2}{|l|}{ Education status } \\
\hline College above & $94(50.0)$ \\
\hline Less than college & $89(47.3)$ \\
\hline Missing & $5(2.7)$ \\
\hline \multicolumn{2}{|l|}{ Employment status } \\
\hline Employed (full time/part time/self) & $58(30.9)$ \\
\hline Unemployed (homemaker/retired) & $89(47.3)$ \\
\hline Medically unable to work & $41(21.8)$ \\
\hline \multicolumn{2}{|l|}{ Medical Insurance } \\
\hline Commercial & $86(45.7)$ \\
\hline Medicare & $43(22.9)$ \\
\hline Medicaid/dual $^{\mathrm{a}}$ & $50(26.6)$ \\
\hline Missing & $9(4.8)$ \\
\hline \multicolumn{2}{|l|}{ Pharmacy insurance } \\
\hline Commercial & $114(60.6)$ \\
\hline Medicare & 35 (18.6) \\
\hline Medicaid/dual & $32(17.0)$ \\
\hline Missing & $7(3.7)$ \\
\hline \multicolumn{2}{|l|}{ Region } \\
\hline Midwest & $40(21.3)$ \\
\hline Northeast & $44(23.4)$ \\
\hline South & $72(38.3)$ \\
\hline West & $31(16.5)$ \\
\hline Missing & $1(0.5)$ \\
\hline
\end{tabular}


Table 2 (continued)

\begin{tabular}{ll}
\hline Characteristic & Total $(N=188)$ \\
\hline Prior stem-cell transplant & $97(51.6)$ \\
No & $91(48.4)$ \\
Yes & \\
Prior treatment administration mode & $119(63.3)$ \\
Oral with or without injectable & $19(10.1)$ \\
Injectable only & $50(26.6)$ \\
None & \\
Current treatment administration mode & $93(49.5)$ \\
All-oral treatment & $95(50.5)$ \\
Injectable with/without oral treatment & \\
Number of agents in current therapy & $135(71.8)$ \\
$1-2$ & $53(28.2)$ \\
$\geq 3$ & $151(80.3)$ \\
Treatment centre & $31(16.5)$ \\
Academic & $6(3.2)$ \\
Community & \\
Other &
\end{tabular}

Data are presented as mean \pm standard deviation or $\mathrm{N}(\%)$ unless otherwise indicated

ECOG PS Eastern Cooperative Oncology Group performance status, $I Q R$ interquartile range

${ }^{a}$ Medicaid/dual refers to enrolees of both Medicare and Medicaid, or Medicare and Commercial insurance

(injectable only vs. any oral therapy) and prior history of transplant status, the global satisfaction scale was not influenced by ECOG PS, race, administration route of current treatment, or other attributes that were tested (Table 3).

\subsection{Patient Work and Activity Impairment}

Table 4 summarizes patient work and activity impairment by treatment administration route. In univariate analyses among employed patients who responded to the WPAI:SHP $(n=55)$, the mean level of impairment while working was significantly higher in patients in the injectable group than in the oral group (38 vs. 20\%; $p=0.037$ ). After adjusting for age, ECOG PS, and prior stem cell transplant history, the level of impairment remained higher in the injectable group than in the oral group, but the difference was not statistically significant (28 vs. $22 \% ; p=0.962$ ). In univariate analyses among all patients with WPAI:SHP responses $(n=155)$, mean level of activity impairment was significantly higher in the injectable group than in the oral group (47 vs. $30 \%$; $p<0.001$ ); after adjustment, a trend towards higher activity impairment was observed in the injectable compared with the oral group ( 43 vs. $34 \% ; p=0.053$ ). There were no significant differences in work time missed and level of overall work impairment between the injectable group and oral

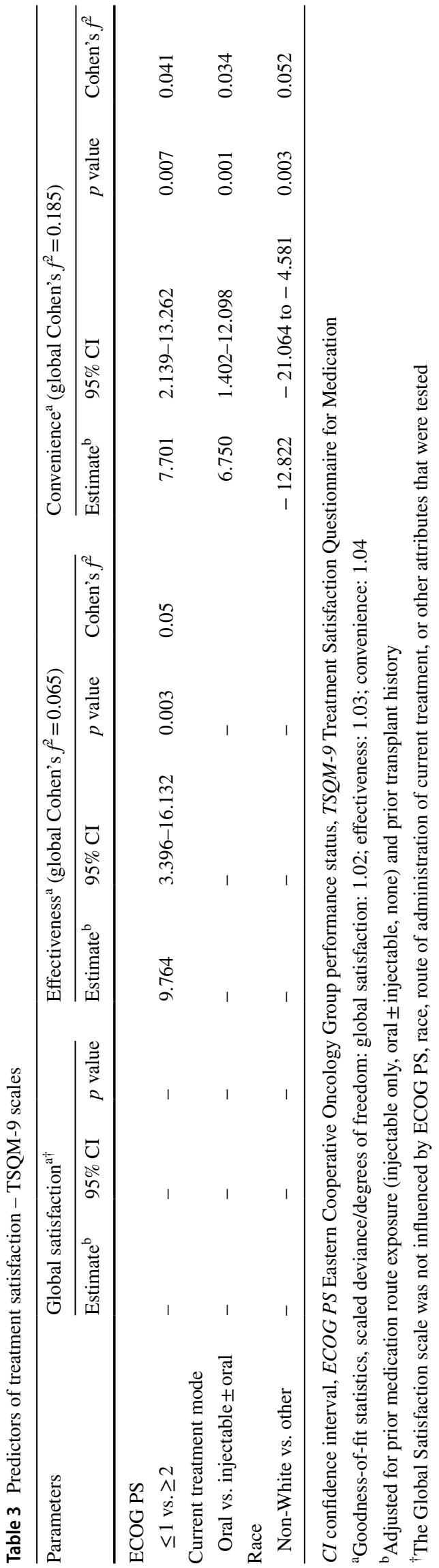


groups after adjustment for age, ECOG PS, and prior stem cell transplant history.

\subsection{Patient and Caregiver Time Burden}

Table 5 summarizes patient and caregiver monthly burden of MM management by treatment administration route. After adjusting for age, ECOG PS, and prior stem cell transplant history, the mean number of treatment visits (3.6 vs. 1.3/ month; $p<0.0001)$, mean time spent at treatment visits (5.4 vs. $3.3 \mathrm{~h} /$ month; $p=0.002$ ), and mean patient ( 8.8 vs. $4.8 \mathrm{~h} /$ month; $p=0.0002)$ and caregiver time burden (6.2 vs. $2.8 \mathrm{~h} /$ month; $p<0.0001)$ were significantly higher in the injectable group than in the oral group.

\subsection{Indirect Costs of Multiple Myeloma Therapy}

Figure 2 shows the unadjusted economic burden of current MM treatment by treatment administration route. Total mean unadjusted monthly costs (\$US442 vs. 167; 2016 values; $p<0.001$ ), mean travel value total (\$US73 vs. $20 ; p<0.001$ ), copay for clinic visits (\$US126 vs. 27 ; $p<0.01$ ), and clinic visit time value for patients (\$US184 vs. $91 ; p<0.05)$ were significantly higher in patients in the
Table 4 Work and activity impairment among patients with newly diagnosed multiple myeloma receiving first-line therapy $^{\mathrm{a}}$

\begin{tabular}{|c|c|c|c|c|}
\hline Characteristic & Total & All oral treatment group & $\begin{array}{l}\text { Injectable with/with- } \\
\text { out oral treatment } \\
\text { group }\end{array}$ & $p$ value $^{\mathrm{b}}$ \\
\hline \multicolumn{5}{|l|}{ WPAI:SHP } \\
\hline \multicolumn{4}{|l|}{$\%$ work time missed ${ }^{\mathrm{c}}$} & 0.091 \\
\hline$n$ included in analyses & 55 & 29 & 26 & \\
\hline Mean \pm SD & $16.3 \pm 28.06$ & $10.0 \pm 17.60$ & $23.3 \pm 35.44$ & \\
\hline Median (IQR) & $0.0(0.0-20.0)$ & $0.0(0.0-16.7)$ & $0.0(0.0-30.8)$ & \\
\hline Adjusted mean $^{\mathrm{d}}$ & & 14.34 & 18.45 & 0.653 \\
\hline \multicolumn{4}{|c|}{$\%$ impairment while working ${ }^{\mathrm{c}}$} & 0.037 \\
\hline$n$ included in analyses & 55 & 29 & 26 & \\
\hline Mean \pm SD & $28.2 \pm 31.10$ & $19.7 \pm 21.29$ & $37.7 \pm 37.45$ & \\
\hline Median (IQR) & $20.0(0.0-50.0)$ & $10.0(0.0-40.0)$ & $25.0(0.0-70.0)$ & \\
\hline Adjusted mean $^{\mathrm{d}}$ & & 21.97 & 28.42 & 0.962 \\
\hline \multicolumn{4}{|c|}{$\%$ overall work impairment ${ }^{\mathrm{c}}$} & 0.078 \\
\hline$n$ included in analyses & 55 & 29 & 26 & \\
\hline Mean \pm SD & $33.5 \pm 33.46$ & $26.0 \pm 26.65$ & $41.9 \pm 38.52$ & \\
\hline Median (IQR) & $25.0(0.0-60.0)$ & $20.0(0.0-40.0)$ & $33.8(0.0-70.0)$ & \\
\hline Adjusted mean $^{\mathrm{d}}$ & & 35.23 & 31.58 & 0.723 \\
\hline \multicolumn{4}{|l|}{$\%$ activity impairment ${ }^{\mathrm{c}}$} & $<0.001$ \\
\hline$n$ included in analyses & 155 & 76 & 79 & \\
\hline Mean \pm SD & $38.8 \pm 29.37$ & $30.1 \pm 27.06$ & $47.1 \pm 29.27$ & \\
\hline Median (IQR) & $40.0(10.0-60.0)$ & $30.0(0.0-50.0)$ & $50.0(20.0-70.0)$ & \\
\hline Adjusted mean $^{\mathrm{d}}$ & & 34.21 & 43.16 & 0.053 \\
\hline
\end{tabular}

The degree to which MM affected absenteeism (work time missed), presenteeism (impairment at work), and work productivity loss (absenteeism plus presenteeism) was measured in employed patients. The degree to which MM affected regular activities (non-work related) was measured in all patients regardless of employment status

$I Q R$ interquartile range, $M M$ multiple myeloma, $N D M M$ newly diagnosed multiple myeloma, $S D$ standard deviation, WPAI:SHP Work Productivity and Activity Impairment Questionnaire: Specific Health Problem

${ }^{a}$ The degree to which MM affected absenteeism (work time missed), presenteeism (impairment at work), and work productivity loss (absenteeism plus presenteeism) was measured in employed patients. The degree to which MM affected regular activities (non-work related) was measured in all patients regardless of employment status

${ }^{\mathrm{b}} p$ values for comparing original mean between oral and injectable \pm oral groups are obtained from Student $t$ test (Satterthwaite method is used if unequal variances); $p$ values for adjusted mean are obtained from general linear model adjusted for age, ECOG PS, and prior stem cell transplant history

${ }^{\mathrm{c}}$ Goodness-of-fit statistics, scaled deviance/degrees of freedom: \% work missed: 1.1; \% impairment while working: $1.1 ; \%$ overall work impairment: $1.1 ; \%$ activity impairment: 1

${ }^{\mathrm{d}}$ Mean for oral vs. injectable \pm oral adjusted for age, ECOG PS, and prior stem cell transplant history 
injectable group than in the oral group. There was no significant difference in clinic visit time value for caregivers between groups. After adjusting for age, ECOG PS, and prior stem cell transplant history, the mean monthly costs in the injectable versus the oral group were \$US482 versus 153 (total costs), \$US130 versus 26 (doctor's visit co-pay), \$US191 versus 88 (patient monthly time value receiving therapy), \$US92 versus 21 (caregiver monthly time value receiving therapy), and $\$$ US 80 versus 20 (total travel value; $p<0.01$ for all comparisons).

\section{Discussion}

This is the first study to identify attributes associated with patient-reported satisfaction with their current therapy for NDMM. Findings showed that worse ECOG PS was independently correlated with lower patient-perceived effectiveness and convenience of their current treatment. Additional predictors of higher patient-perceived convenience with treatment included White race and oral administration route of current therapy. We also evaluated patient work and activity impairment and patient and caregiver treatment-related time burden and indirect costs of NDMM therapy. The use of therapies containing an injectable agent was associated with increased activity impairment, increased time burden, and higher indirect costs of MM therapy for patients and caregivers compared with solely orally administered therapies.

This study used the TSQM-9 to investigate satisfaction with current treatment in patients with NDMM. The TSQM-9 is a general measure of patients' satisfaction with medication. Findings revealed that, in patients with NDMM, ECOG PS was the most important predictor of patientperceived effectiveness of current treatment. Interestingly, ECOG PS was not associated with global satisfaction with MM treatment. Association between clinical variables and treatment satisfaction has been reported in studies of patients with other malignancies. In US patients with breast cancer, physician-reported ECOG PS was associated with expectations for therapy [30]. In older and younger patients included in the University of North Carolina Health Registry Cancer Survivorship Cohort, better self-reported ECOG PS was associated with higher satisfaction with care [31].

In this study, patients identifying as non-White race reported decreased patient-perceived convenience of their current treatment compared with White patients. Given the
Table 5 Patient and caregiver monthly burden of multiple myeloma management by route of administration

\begin{tabular}{|c|c|c|c|c|}
\hline Variable & Total $(N=188)$ & $\begin{array}{l}\text { All-oral treatment } \\
(N=93)\end{array}$ & $\begin{array}{l}\text { Injectable with/without oral } \\
\text { treatment }(N=95)\end{array}$ & $p$ value $^{\mathrm{a}}$ \\
\hline \multicolumn{4}{|c|}{ Number of treatment visits/month ${ }^{b}$} & $<0.0001$ \\
\hline Mean \pm SD & $2.5 \pm 2.93$ & $1.2 \pm 2.73$ & $3.8 \pm 2.56$ & \\
\hline Median (IQR) & $2.0(1.0-4.0)$ & $1.0(0.0-1.0)$ & $4.0(2.0-5.0)$ & \\
\hline Adjusted mean ${ }^{\mathrm{c}}$ & & 1.29 & 3.61 & $<0.0001$ \\
\hline \multicolumn{4}{|c|}{ Average time spent at treatment visits, $\mathrm{h} /$ month $^{\mathrm{b}}$} & 0.026 \\
\hline Mean \pm SD & $3.4 \pm 7.48$ & $2.2 \pm 9.15$ & $4.6 \pm 5.15$ & \\
\hline Median (IQR) & $1.4(0.1-4.0)$ & $0.4(0.0-2.0)$ & $3.0(1.0-6.0)$ & \\
\hline Adjusted mean ${ }^{\mathrm{c}}$ & & 3.33 & 5.39 & 0.002 \\
\hline \multicolumn{4}{|c|}{ Patient time burden, $\mathrm{h} / \mathrm{month}^{\mathrm{b}}$} & 0.020 \\
\hline Mean \pm SD & $5.8 \pm 11.48$ & $3.8 \pm 14.45$ & $7.7 \pm 7.07$ & \\
\hline Median (IQR) & $2.9(1.1-7.5)$ & $1.4(0.0-3.3)$ & $5.0(2.8-10.0)$ & \\
\hline Adjusted mean ${ }^{\mathrm{c}}$ & & 4.78 & 8.76 & 0.0002 \\
\hline \multicolumn{4}{|c|}{ Caregiver time burden, $\mathrm{h} /$ month $^{\mathrm{b}}$} & 0.173 \\
\hline Mean \pm SD & $3.3 \pm 11.06$ & $2.2 \pm 14.29$ & $4.4 \pm 6.41$ & \\
\hline Median (IQR) & $0.0(0.0-3.4)$ & $0.0(0.0-0.0)$ & $2.6(0.0-5.7)$ & \\
\hline Adjusted mean ${ }^{\mathrm{c}}$ & & 2.83 & 6.18 & $<0.0001$ \\
\hline
\end{tabular}

The value of patient time was applied to all patients regardless of employment type. All data pertaining to caregivers were obtained indirectly from patient reports

ECOG PS Eastern Cooperative Oncology Group performance status, $I Q R$ interquartile range, $S D$ standard deviation

${ }^{\mathrm{a}} p$ values are for comparison between oral and injectable groups; student's $t$ test for continuous variables and Chi-squared tests for categorical variables; the missing categories for each categorical variable are excluded from the Chi-squared tests

${ }^{\mathrm{b}}$ Goodness-of-fit statistics, scaled deviance/degrees of freedom: number of treatment visits: 1.95; average time spent at treatment visits: 1.15; patient time burden, hours: 1.16; caregiver time burden: 1.19

${ }^{\mathrm{c}}$ Mean for oral vs. injectable \pm oral adjusted for age, ECOG PS, and prior stem cell transplant history 
Fig. 2 Monthly mean cost for multiple myeloma treatment per patient per route of administration. Error bars represent the $95 \%$ confidence intervals. $* p<0.05, * * p<0.01$, $* * * p<0.001$

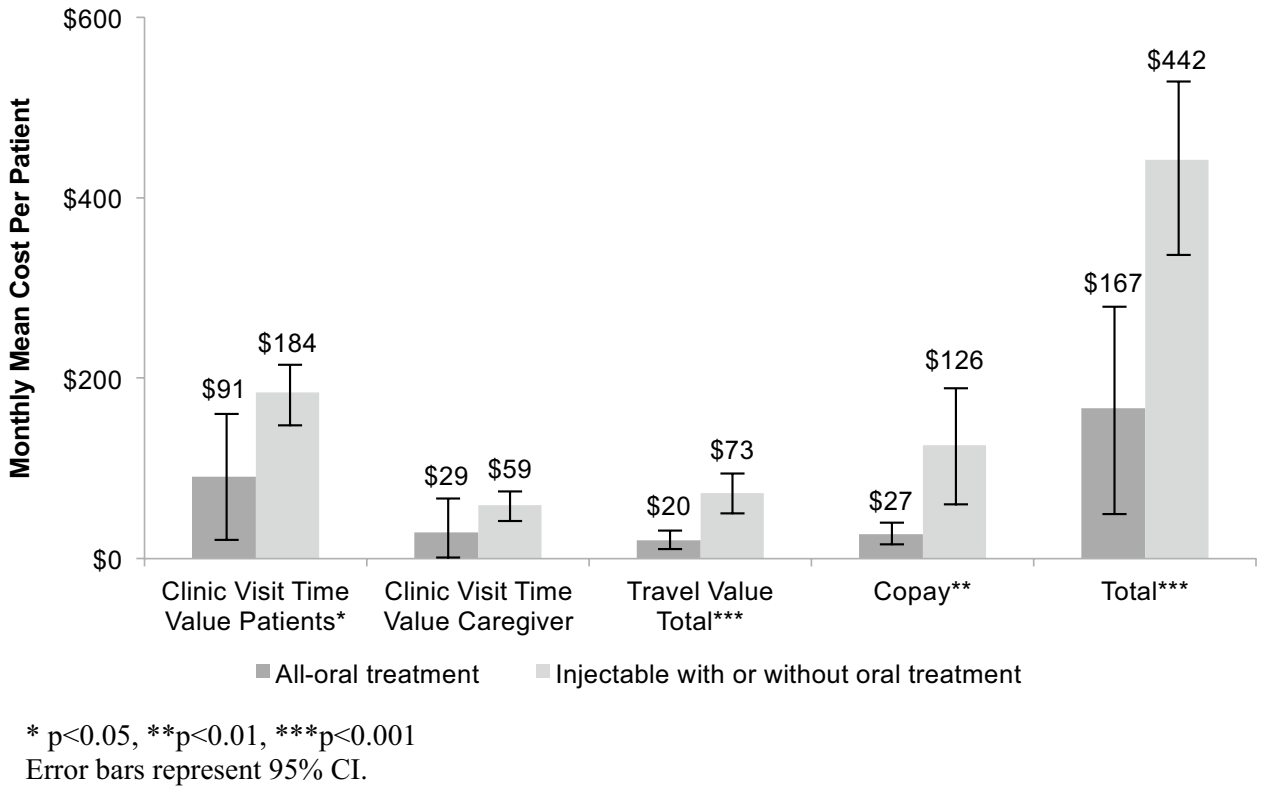

small number of patients, with 18 non-White responders (15 identifying as African American), this finding should be interpreted with caution; however, it is interesting, as racial disparities in the incidence of $\mathrm{MM}$ and access to medical care exist. A population-based study using nine Surveillance, Epidemiology, and End Results Program (SEER) registries, including 5798 African Americans and 28,939 White patients with MM diagnosed between 1973 and 2005, and followed through 2006, showed the incidence of MM among African Americans was twice that among Whites [32]. The potential racial disparity in patient satisfaction with MM treatments identified in this study warrants further investigation of individual patient preferences in treatment selection.

Reports have shown that treatment has a substantial impact on work productivity and functioning in patients with MM [33]. One study in patients with MM who had received intensive treatment showed that only $33 \%$ of patients were employed after treatment compared with $66 \%$ before diagnosis [16]. In the present study, one-fifth of patients reported inability to work for medical reasons, and injectable treatment administration route was associated with a trend towards higher activity impairment in patients with NDMM. A regimen that included injectable therapy was correlated with more work time missed and impairment while working, but the results did not reach statistical significance in adjusted analyses because of the small sample sizes in the subgroup of patients who were employed. Similar findings were reported in a study of US patients with MM identified from the Lightspeed GMI global panel and its partners, which showed that patients on an all-oral regimen reported significantly lower overall work impairment than those on an injectable regimen, primarily due to decreased impairment while at work [34]. Future research is needed to determine the relationship between adherence, treatment modality, and work/activity impairment in patients with NDMM.

Evidence suggests that patients with $\mathrm{MM}$ are concerned about the personal financial impact associated with their disease [16]. Some patients with MM report out-of-pocket expenses amounting to $36 \%$ of their income in the first treatment year [16]. Patients with MM who had received at least 3 months of treatment at a US tertiary academic medical centre indicated that they applied for financial assistance (36\%), used their savings to pay for myeloma treatment (46\%), and borrowed money to pay for medications $(21 \%)$ [9]. In the present study, we did not evaluate the out-ofpocket expenditures for medications, but the total monthly costs that included travel value total, outpatient visit copays, and clinic visit time value were significantly lower in patients treated with an all-oral regimen compared with a regimen that included injectable with or without oral therapy. Consistent with these findings, another study reported that patients with MM using an oral-only regimen reported fewer clinic visits in the past 3 months, lower out-of-pocket costs for these visits, and less time spent at appointments related to $\mathrm{MM}$ treatments in the past month than did those receiving an injectable regimen [34]. These findings suggest that the personal financial needs of patients with NDMM should be considered during treatment decision making.

\subsection{Limitations}

This study was associated with several limitations. First, the sample size was small, and a potential existed for selection bias in treatment administration to individual patients, as treatment assignment was not randomized. Second, data were collected through patient self-report, which may have 
been confounded by the lack of clinical validation of patient-, disease-, and treatment-related factors and recall/confirmation bias. Third, this is the first study to use the TSQM-9 scale to investigate satisfaction with current treatment for patients with NDMM. This scale provides a general measure of patients' satisfaction with medication. It was previously validated across different types of medication and diverse patient populations, including patients with cancer; however, it has not been validated in NDMM [20]. Fourth, the patients included in this study may not have been representative of the general NDMM patient population. Patients in online communities are usually a self-selected sample of mostly female, educated patients, and the majority of patients in the current study were treated at an academic centre. In contrast to our study cohort, which included younger patients with overrepresentation of female patients (mean \pm SD age $61 \pm 8.92$ years; $61 \%$ female), a real-world data analysis of the demographic and comorbidity characteristics among a representative sample of 8239 patients with NDMM in the USA reported a mean \pm SD age of $66.2 \pm 11.3$ years at diagnosis (51.8\% female patients) [35]. Fifth, financial analyses were performed for the USA, and findings may not be generalizable to other countries. Finally, this was a crosssectional study reflecting the patient experience of a month of the NDMM treatment process, which may require a longer follow-up with a larger sample across multiple regimens to increase the generalizability of the results $[3,36]$.

\section{Conclusion}

This study showed that a better ECOG PS was associated with higher patient-perceived effectiveness and convenience of their current NDMM treatment. The use of current alloral therapy was associated with increased patient-reported treatment convenience. The data suggest that an oral regimen is associated with a higher level of convenience for patients with NDMM, decreased activity impairment, decreased time burden, and fewer indirect costs than an injectable regimen. The use of oral drugs among patients with NDMM could reduce the time burden associated with their disease. This study, albeit based on a small sample, identified a notable racial disparity in patient satisfaction with MM treatments, which should be evaluated in further research of individual patient preferences in treatment selection. Findings from the present study may better inform treatment decision making in NDMM.

Acknowledgements The authors wish to acknowledge medical writing support provided by Jane Kondejewski, PhD. Funding for medical writing support was provided by Millennium Pharmaceuticals, Inc., a wholly owned subsidiary of Takeda Pharmaceutical Company Limited, Cambridge, MA, USA.
Author Contributions All authors contributed equally to the study design, data analysis, and manuscript development and approval.

Data Availability The data that support the findings of this study are available from PatientsLikeMe ${ }^{\circledR}$, but restrictions apply to the availability of these data, which were used under license for the current study, and so are not publicly available. Data are however available from the authors upon reasonable request and with permission of PatientsLikeMe ${ }^{\circledR}$

\section{Compliance with Ethical Standards}

Conflicts of Interest RR is an employee of the Rocky Mountain Cancer Centers, received funding from the study sponsor, and has received consulting honoraria from or has membership on the following entities' board of directors or advisory committees: Amgen, Celgene, Takeda, Janssen. JB, SC, and DR are employees of Millennium Pharmaceuticals, Inc. PH was an employee of Millennium Pharmaceuticals, Inc. at the time of the study. PD was an employee of PatientsLikeMe at the time of the study and received funding from the study sponsor. $\mathrm{MH}$, ML, and CC are employees of PatientsLikeMe and have received funding from the study sponsor.

Ethical Approval The study received ethical approval from the New England Institutional Review Board. Patients provided informed consent electronically prior to completing the survey. Respondents were not remunerated for their participation.

Funding This study was funded by Millennium Pharmaceuticals, Inc., a wholly owned subsidiary of Takeda Pharmaceutical Company Limited, Cambridge, MA, USA.

Open Access This article is distributed under the terms of the Creative Commons Attribution-NonCommercial 4.0 International License (http://creativecommons.org/licenses/by-nc/4.0/), which permits any noncommercial use, distribution, and reproduction in any medium, provided you give appropriate credit to the original author(s) and the source, provide a link to the Creative Commons license, and indicate if changes were made.

\section{References}

1. SEER. Cancer stat facts: myeloma. National Cancer Institute. 2018. https://seer.cancer.gov/statfacts/html/mulmy.html. Accessed 5 Apr 2019.

2. Röllig C, Knop S, Bornhäuser M. Multiple myeloma. Lancet. 2015;385(9983):2197-208.

3. Chim CS, Kumar SK, Orlowski RZ, et al. Management of relapsed and refractory multiple myeloma: novel agents, antibodies, immunotherapies and beyond. Leukemia. 2018;32(2):252-62.

4. Kumar SK, Callander NS, Alsina N, et al. NCCN Guidelines ${ }^{\circledR}$ insights: multiple myeloma, Version 3.2018. J Natl Compr Canc Netw. 2018;16(1):11-20.

5. Al-Abri R, Al-Balushi A. Patient satisfaction survey as a tool towards quality improvement. Oman Med J. 2014;29(1):3-7.

6. Efficace F, Cottone F, Abel G, et al. Patient-reported outcomes enhance the survival prediction of traditional disease risk classifications: an international study in patients with myelodysplastic syndromes. Cancer. 2018;124(6):1251-9.

7. Eek D, Krohe M, Mazar I, et al. Patient-reported preferences for oral versus intravenous administration for the treatment 
of cancer: a review of the literature. Patient Prefer Adherence. 2016;10:1609-21.

8. Gupta D, Rodeghier M, Lis CG. Patient satisfaction with service quality as a predictor of survival outcomes in breast cancer. Support Care Cancer. 2014;22(1):129-34.

9. Huntington SF, Weiss BM, Vogl DT, et al. Financial toxicity in injured patients with multiple myeloma: a cross-sectional pilot study. Lancet Haematol. 2015;2(10):e408-16.

10. Schott S, Schneeweiss A, Reinhardt J, et al. Acceptance of oral chemotherapy in breast cancer patients - a survey study. BMC Cancer. 2011;11:129.

11. Gornas M, Szczylik C. Oral treatment of metastatic breast cancer with capecitabine: what influences the decision-making process? Eur J Cancer Care (Engl). 2010;19(1):131-6.

12. Beusterien K, Grinspan J, Kuchuk I, et al. Use of conjoint analysis to assess breast cancer patient preferences for chemotherapy side effects. Oncologist. 2014;19(2):127-34.

13. Doyle C, Lennox L, Bell D. A systematic review of evidence on the links between patient experience and clinical safety and effectiveness. BMJ Open. 2013;3(1):e001570.

14. Hari P, Romanus D, Palumbo A, et al. Prolonged duration of therapy is associated with improved survival in patients treated for relapsed/refractory multiple myeloma in routine clinical care in the United States. Clin Lymphoma Myeloma Leuk. 2018;18(2):152-60.

15. Burnette BL, Dispenzieri A, Kumar S, et al. Treatment trade-offs in myeloma: a survey of consecutive patients about contemporary maintenance strategies. Cancer. 2013;119(24):4308-15.

16. Goodwin JA, Coleman EA, Sullivan E, et al. Personal financial effects of multiple myeloma and its treatment. Cancer Nurs. 2013;36(4):301-8.

17. Bharmal M, Payne K, Atkinson MJ, et al. Validation of an abbreviated Treatment Satisfaction Questionnaire for Medication (TSQM-9) among patients on antihypertensive medications. Health Qual Life Outcomes. 2009;7(1):36.

18. Basch E, Artz D, Dulko D, et al. Patient online self-reporting of toxicity symptoms during chemotherapy. J Clin Oncol. 2005;23(15):3552-61.

19. Reilly MC, Zbrozek AS, Dukes EM. The validity and reproducibility of a work productivity and activity impairment instrument. Pharmacoeconomics. 1993;4(5):353-65.

20. Atkinson MJ, Sinha A, Hass SL, et al. Validation of a general measure of treatment satisfaction, the Treatment Satisfaction Questionnaire for Medication (TSQM), using a national panel study of chronic disease. Health Qual Life Outcomes. 2004;2:12.

21. Oken MM, Creech RH, Tormey DC, et al. Toxicity and response criteria of the Eastern Cooperative Oncology Group. Am J Clin Oncol. 1982;5(6):649-55
22. Cohen JE. Statistical Power Analysis for the Behavioral Sciences. Hillsdale: Lawrence Erlbaum Associates, Inc.; 1988.

23. Bureau of Labor Statistics (BLS) National Average Wage all Occupations. 2016. https://www.bls.gov/oes/2016/may/oes_nat. htm. Accessed 5 Apr 2019.

24. National Conference of state legislatures (NCSL). 2017. http:// www.ncsl.org/research/labor-and-employment/state-minimumwage-chart.aspx. Accessed 8 Apr 2019.

25. Bureau of Labor Statistics (BLS) Mean Home Health Aide Wage. 2016. https://www.bls.gov/ooh/healthcare/home-health-aides-andpersonal-care-aides.htm. Accessed 5 Apr 2019.

26. Cost of Care Survey. 2016. https://www.genworth.com/about-us/ industry-expertise/cost-of-care.html. Accessed 8 Apr 2019.

27. Internal Revenue Service (IRS) mileage rates. 2017. https://www. irs.gov/newsroom/2017-standard-mileage-rates-for-business-andmedical-and-moving-announced. Accessed 8 Apr 2019.

28. Public transportation Fact book, 67th edition, February 2017. American Public Transportation Association.

29. TaxiFareFinder. https://www.taxifarefinder.com/main. php?city=AnyUS. Accessed 5 Apr 2019.

30. Trask PC, Tellefsen C, Espindle D, et al. Clinical variables are associated with treatment satisfaction in patients with breast, lung, and colorectal cancer. J Clin Oncol. 2007;25(18Suppl):6608.

31. Mariano C, Hanson LC, Deal AM, et al. Healthcare satisfaction in older and younger patients with cancer. J Geriatr Oncol. 2016;7(1):32-8

32. Waxman AJ, Mink PJ, Devesa SS, et al. Racial disparities in incidence and outcome in multiple myeloma: a population-based study. Blood. 2010;116(25):5501-6.

33. Gupta S, Abouzaid S, Liebert R, et al. Assessing the effect of adherence on patient-reported outcomes and out of pocket costs among patients with multiple myeloma. Clin Lymph Myeloma Leuk. 2018;18(3):210-8.

34. Gupta S, Abouzaid S, Liebert R, et al. Assessing the effect of injectable or oral routes of treatment administration on patientreported outcomes and out-of-pocket costs among patients with multiple myeloma. Blood. 2016;128(22):5931. http://www.blood journal.org/content/128/22/5931. Accessed 30 Oct 302018.

35. Chen YJ, De AP, Cong Z, Aggarwal SK, et al. Demographic and comorbidity characteristics of newly diagnosed multiple myeloma patients in the United States: a real world data analysis. Blood. 2014;124:1301.

36. Boudreault JS, Touzeau C, Moreau P. Triplet combinations in relapsed/refractory myeloma: update on recent phase 3 trials. Expert Rev Hematol. 2017;10(3):207-15. 